\title{
Endoscopic Ultrasound with Bronchoscope-Guided Fine Needle Aspiration for the Diagnosis of Paraesophageally Located Lung Lesions
}

\author{
Ida Skovgaard Christiansen ${ }^{a, b} \quad$ Jolanda Corina Kuijvenhoven ${ }^{\mathrm{e}} \quad$ Uffe Bodtger $^{\mathrm{a}-\mathrm{c}}$ \\ Therese Maria Henriette Naur $^{\text {b, d }}$ Khaliq Ahmad $^{\text {b Jatinder Singh Sidhu }}{ }^{b}$ Rafi Nessar $^{a}$ \\ Goran Nadir Salih $^{\mathrm{a}}$ Asbjørn Høegholm ${ }^{\mathrm{b}}$ Jouke Tabe Annema ${ }^{\mathrm{e}}$ Paul Frost Clementsen ${ }^{\mathrm{a}, \mathrm{c}}$ \\ a Department of Internal Medicine, Zealand University Hospital, Roskilde, Denmark; ${ }^{b}$ Department of Respiratory \\ Medicine, Næstved Hospital, Næstved, Denmark; ' Institute of Regional Health Research, University of Southern Denmark, \\ Odense, Denmark; ${ }^{\mathrm{d} C o p e n h a g e n ~ A c a d e m y ~ f o r ~ M e d i c a l ~ E d u c a t i o n ~ a n d ~ S i m u l a t i o n ~(C A M E S), ~ R i g s h o s p i t a l e t, ~ U n i v e r s i t y ~}$

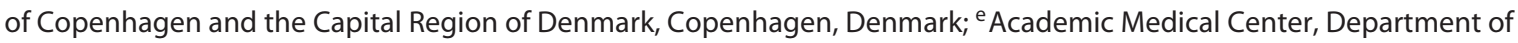 \\ Respiratory Medicine, University of Amsterdam, Amsterdam, The Netherlands
}

\section{Keywords}

Lung cancer · Diagnosis · Endobronchial ultrasound ·

Esophageal ultrasound

\section{Abstract}

Background: Diagnosing centrally located lung tumors without endobronchial abnormalities and not located near the major airways is a diagnostic challenge. Tumors near or adjacent to the esophagus can be aspirated and detected with esophageal ultrasound (EUS) using gastrointestinal endoscopes. Objective: To assess the feasibility and diagnostic yield of endoscopic ultrasound with bronchoscope-guided fine needle aspiration (EUS-B-FNA) in paraesophageally located lung tumors and its added value to bronchoscopy and endobronchial ultrasound (EBUS). Methods: Retrospective, multicenter international study (from January 1, 2015 until January 1,2018 ) of patients with suspected lung cancer, undergoing bronchoscopy, EBUS, and endoscopic ultrasound bronchoscopy (EUS-B) in one session by a single operator (pulmonologist), in whom the primary lung tumor was detected and aspirated by EUS-B. In the absence of malignancy following endoscopy, transthoracic ultrasound needle aspi- ration, clinical and radiological follow-up of at least 6 months was performed. The yield and sensitivity of EUS-B-FNA and its added value to bronchoscopy and EBUS was assessed. $\boldsymbol{R} \boldsymbol{e}$ sults: 58 patients were identified with the following diagnosis: non-small-cell lung cancer $(n=43)$, small-cell lung cancer $(n=6)$, mesothelioma $(n=2)$, metastasis $(n=1)$, nonmalignant $(n=6)$. The yield and sensitivity of EUS-B-FNA for detecting lung cancer was $90 \%$. In 26 patients (45\%), the intrapulmonary tumor was exclusively detected by EUS-B. Adding EUS-B to conventional bronchoscopy and EBUS increased the diagnostic yield for diagnosing lung cancer in paraesophageally located lung tumors from 51 to $91 \%$. No EUSB-related complications were observed. Conclusion: EUS-BFNA is a feasible and safe technique for diagnosing centrally located intrapulmonary tumors that are located near or adjacent to the esophagus. EUS-B should be considered in the same endoscopy session following nondiagnostic bronchoscopy and EBUS.

(C) 2018 The Author(s)

Published by S. Karger AG, Basel

Ida Skovgaard Christiansen and Jolanda Corina Kuijvenhoven contributed equally to this work.

\begin{tabular}{|c|c|}
\hline KARGER & $\begin{array}{l}\text { (c) } 2018 \text { The Author(s) } \\
\text { Published by S. Karger AG, Basel }\end{array}$ \\
\hline $\begin{array}{l}\text { E-Mail karger@karger.com } \\
\text { www.karger.com/res }\end{array}$ & $\begin{array}{l}\text { This article is licensed under the Creative Commons Attribution- } \\
\text { NonCommercial-NoDerivatives } 4.0 \text { International License (CC BY- } \\
\text { NC-ND) (http://www.karger.com/Services/OpenAccessLicense). } \\
\text { Usage and distribution for commercial purposes as well as any dis- } \\
\text { tribution of modified material requires written permission. }\end{array}$ \\
\hline
\end{tabular}

Jouke T. Annema, MD, PhD

Academic Medical Centre, University of Amsterdam

Meibergdreef 9

NL-1105 AZ Amsterdam (The Netherlands)

E-Mail j.t.annema@amc.uva.nl 


\section{Introduction}

Lung cancer is the leading cause of cancer-related mortality worldwide [1]. In patients with suspected lung cancer, a tissue diagnosis is crucial to establish a definite diagnosis. However, for patients presenting with a centrally located lung tumor without endobronchial abnormalities and not near the major airways, obtaining a tissue diagnosis is a diagnostic challenge.

In routine practice, flexible bronchoscopy with its associated procedures (endobronchial biopsy, brushing, and washing) is performed, especially in case of a visible endobronchial tumor. However, frequently, no endobronchial abnormalities are visible and in these situations, the diagnostic yield by standard bronchoscopic techniques is low [2-6]. Guidance techniques (radial endobronchial ultrasound [EBUS]/fluoroscopy, navigation) can be helpful in peripherally located lung lesions in case an airway leads to the tumor but often do not contribute to the diagnostic yield of these specific central lesions $[7,8]$.

If the tumor is located near or adjacent to the large airways, endobronchial ultrasound transbronchial needle aspiration (EBUS-TBNA) is a useful and safe procedure to obtain a tissue diagnosis $[9,10]$.

With gastrointestinal endoscopes it has been proven that lung tumors located near or adjacent to the esophagus can be detected and aspirated with esophageal ultrasound (EUS) [11]. However, this technique is not commonly available in most pulmonary practices. Current lung cancer staging guidelines recommend endoscopic ultrasound bronchoscopy (EUS-B) (using the EBUS scope in the esophagus) for mediastinal staging, because this is complementary to EBUS for mediastinal nodal staging [12-15]. EBUS and EUS-B are also suggested in this guideline for the analysis of lung tumors in patients with a centrally located lung tumor that are not visible with conventional bronchoscopy, provided that the tumor is located immediately adjacent to the larger airways (EBUS-TBNA) or esophagus (EUS-B/ EUS) [16].

To date, however, there is only limited evidence of the value of endoscopic ultrasound with bronchoscope-guided fine needle aspiration (EUS-B-FNA) for obtaining a tissue diagnosis in centrally located lung tumors $[17,18]$. Therefore, we conducted this study to assess the feasibility and diagnostic yield of EUS-B in paraesophageally located lung tumors and its added value to bronchoscopy and EBUS.

\section{Methods}

Study Design and Patients

This is a retrospective multicenter international study undertaken in the Naestved Hospital, Naestved, Denmark (Department of Internal Medicine), the Zealand University Hospital, Roskilde, Denmark (Department of Internal Medicine), and in the Academic Medical Center (Department of Respiratory Medicine, University of Amsterdam), Amsterdam, the Netherlands, during the period of January 1, 2015 until January 1, 2018. Patient data were retrieved for the various endosonography databases in the 3 hospitals based on the following criteria: patients who underwent an EUS-B-FNA to obtain a tissue diagnosis of an intrapulmonary lesion for suspected lung cancer and also routinely underwent bronchoscopy and EBUS.

\section{Patient Selection}

All patients in whom the paraesophageally located lung tumor was detected by EUS-B and sampled were identified. The presented case is an example of the patient selection (Fig. 1). All CT and PET-CT imaging, bronchoscopy, EBUS, EUS-B reports, cytopathological reports and follow-up data were collected. Also, complications of EUS-B were retrieved.

\section{Study Endpoints}

The primary endpoints of this study were to assess the diagnostic yield of EUS-B and its added value to conventional bronchoscopy and EBUS for obtaining a tissue diagnosis of centrally located lung tumors located near or adjacent to the esophagus.

The secondary endpoints were the adequacy of the tissue samples and sensitivity of EUS-B for the diagnosis of a centrally located lung tumor, the feasibility of EUS-B, and EUS-B-related complication for diagnosing an intrapulmonary tumor.

\section{Definitions of Sample Adequacy, Yield, and Sensitivity}

Biopsies were judged to be adequate when containing material sufficient for cytopathological evaluation. Samples in which cytopathological evaluation showed malignancy were considered to be true positive.

When cytopathological evaluation of EUS-B-FNA samples showed no malignancy, the EUS-B diagnosis of the lung lesion was confirmed with at least 6 months of follow-up with clinical course and/or CT. For the calculation of the yield and sensitivity of malignancy, samples with a nonmalignant diagnosis without followup were assumed to be false negative in the analyses (worst case scenario).

The diagnostic yield was defined as the number of samples in which EUS-B-FNA provided a correct diagnosis relative to the total number of samples assessed with EUS-B-FNA [11].

Sensitivity of malignancy was defined as the number of samples in which EUS-B-FNA diagnosed any malignancy relative to the total number of samples where the targeted intrapulmonary tumor turned out to be malignant [11].

\section{The EUS-B Procedure}

Procedures were performed under conscious sedation using midazolam/fentanyl or propofol sedation. Following a conventional bronchoscopy, systematic EBUS was performed according to EBUS-STAT [19]. 

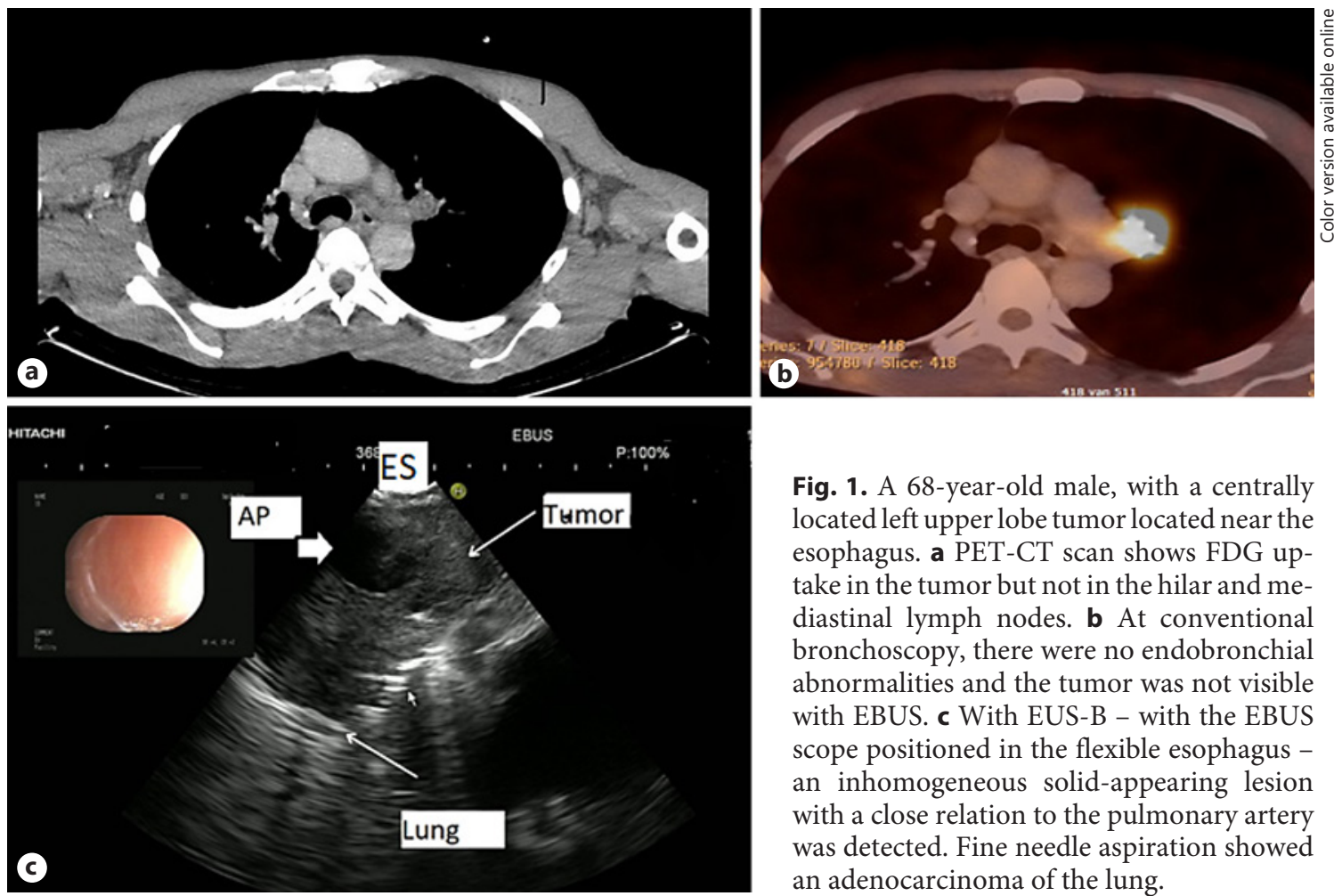

Fig. 1. A 68-year-old male, with a centrally located left upper lobe tumor located near the esophagus. a PET-CT scan shows FDG uptake in the tumor but not in the hilar and mediastinal lymph nodes. b At conventional bronchoscopy, there were no endobronchial abnormalities and the tumor was not visible with EBUS. c With EUS-B - with the EBUS scope positioned in the flexible esophagus an inhomogeneous solid-appearing lesion with a close relation to the pulmonary artery was detected. Fine needle aspiration showed an adenocarcinoma of the lung.

Following EBUS, the EUS-B procedure will be discussed in more detail: for EUS-B-FNA, a flexible EBUS endoscope (Olympus BF-UC180F or UC 180F, Olympus Medical Systems Europe, Ltd., Hamburg, Germany, or Pentax EB-1970 UK, Olympus BFUC180F) was used.

The EBUS endoscope was introduced into the esophagus by retracting the EBUS scope from the trachea to a level just above the vocal cords and from this position turning it slightly to the left and the back of the patient and advancing it into the esophagus under gentle pressure while the patient was encouraged to swallow (in case of mild sedation). The endoscope was advanced carefully till the liver was visualized on ultrasound imaging.

A structured EUS assessment was performed using the esophageal assessment tool (EUS-AT) with 6 landmarks identified in this order: the liver, the abdominal aorta, the left adrenal gland, lymph node station 7 , station $4 \mathrm{~L}$ and $4 \mathrm{R}$. This validated and systematic assessment tool is specifically developed for the examination of lung cancer patients [20]. Following the identification of the intrapulmonary tumor, aspirates were performed using a $21-\mathrm{G}$ or a $22-\mathrm{G}$ needle (22 Gauge Olympus ViziShot and ViziShot 2, Olympus Medical Systems Europe, Ltd., Hamburg, Germany, or a 21- to 22-G COOK needle). When the needle was placed in the lesion under ultrasonic guidance, the stylet was removed, and suction was applied, or the stylet was removed using the slow pull technique. At least two samples were taken. The aspirates were processed for both cytological smears and cellblock analysis. A chest-X-ray was not performed routinely after the procedure.

EUS-B-FNA for the Diagnosis of

Paraesophageally Located Lung Lesions

\section{Statistics}

Data were nonparametric and presented with median and range. Data were processed using SPSS (IBM SPSS Statistics, version 22. Chicago, IL, USA).

\section{Ethics}

This retrospective analysis was conducted in accordance with the amended Declaration of Helsinki and publication of the data was approved by the Data Protection Agency in Denmark and the medical ethics committee in the Netherlands.

\section{Results}

Fifty-eight patients were identified who underwent EUS-B-FNA for diagnosing an intrapulmonary tumor between January 1, 2015 and January 1, 2018. Patient characteristics are shown in Table 1. Of the 58 patients, 34 were female $(59 \%)$ with a median age of 78 years. The aspirated lesions were localized in all lobes, with a median size of $55 \mathrm{~mm}$. No EUS-B-related complications were observed.

Final diagnoses were: non-small cell lung cancer $(n=$ 43; adenocarcinoma $n=26$; squamous cell carcinoma $n=$ 12; non-small cell lung cancer not otherwise specified $n=$ $5)$, small cell lung cancer $(n=6)$, malignant mesothelioma 
Table 1. Baseline characteristics of the patients included in the analysis and final diagnosis after complete workup

\begin{tabular}{ll}
\hline Patients, $n$ & 58 \\
Median age, years & $78(41-90)$ \\
Gender & $24(41)$ \\
Male & $34(59)$ \\
Female & $20(34)$ \\
Localization of the lung tumor & $6(10)$ \\
LUL & $18(31)$ \\
LLL & $1(2)$ \\
RUL & $13(22)$ \\
RML & $55(7-120)$ \\
RLL & NSCLC, $n=43$ \\
Median tumor size long axis, mm & (adenocarcinoma $n=26$, squamous cell carcinoma $n=12$, NSCLC-NOS $n=5)$ \\
Final diagnosis after complete workup & SCLC, $n=6$ \\
& Malignant mesothelioma, $n=2$ \\
& Metastasis from an extrapulmonary tumor, $n=1$ \\
& Nonmalignant, $n=6$ \\
& (unspecific lesion $n=1$, infection $n=5)$ \\
EUS-B-related complications & none
\end{tabular}

Figures in parentheses are percentages or ranges. LUL, left upper lobe; LLL, left lower lobe; RUL, right upper lobe; RML, right middle lobe; RLL, right lower lobe; NSCLC, non-small-cell lung cancer; NOS, not otherwise specified; SCLC, small-cell lung cancer.

$(n=2)$, and metastasis from extrapulmonary cancer $(n=$ 1 , anal squamous cell carcinoma). In 6 patients, the diagnosis was nonmalignant (unspecific lesion; $n=1$, infectious cause $n=5$; Table 1 ).

Fifty-three patients (91\%) had a bronchoscopy and EBUS-TBNA was performed in the same session as EUSB. Five patients did not undergo bronchoscopy or EBUS due to respiratory problems $(n=2)$ or the endobronchial procedure was expected to be of little consequence in obtaining a tissue diagnosis $(n=3)$ due to the anatomical position of the lung lesion.

At bronchoscopy, in 14 patients (24\%), the tumor was visualized and biopsied. All these samples showed a malignancy. The diagnostic yield of the bronchoscopy was 26\%. Another 14 patients (22\%) underwent an EBUS. Thirteen of the samples obtained with EBUS were adequate for cytopathological evaluation; in 11 cases, the samples showed malignancy, in 2 patients the aspirates were nonmalignant ( 1 showed a necrotizing granulomatous inflammation consistent with tuberculosis and 1 showed a reactive and inflammatory changes at cytopathology with a clinical picture of pneumonia and full regression on antibiotic treatment). The diagnostic yield of EBUS alone was 25\%. Adding EBUS to the bronchoscopy raised the diagnostic yield from 26 to $51 \%$. In 1 (2\%) case, the tumor could be visualized but not biopsied with EBUS-TBNA.

All 58 patients underwent EUS-B; of these, 52 were diagnostic and 47 were diagnosed to be malignant. In 26 patients (45\%), the tumor was exclusively visualized and biopsied with EUS-B-FNA. The diagnostic yield for EUS$\mathrm{B}$ alone was $90 \%$. Combining bronchoscopy, EBUS, and EUS-B resulted in a diagnostic yield of $91 \%$ (Fig. 2).

Of all the 58 samples included in the analysis, 55 (95\%) samples were adequate. Of these, $85 \%(n=47)$ were malignant and $15 \%(n=8)$ were nonmalignant. Of these, 4 cases had a clinical and radiological follow-up for at least 6 months, the causes were infectious, and 1 case had the results confirmed in repeated EUS-B-FNA and follow-up CT (2 samples with reactive and inflammatory changes at cytopathology with confirmed microbiologic agents or full regression on antibiotic treatment, 2 samples showed necrotizing granulomatous inflammation consistent with tuberculosis and one sample showed inflammatory cells confirmed at re-examination). These 5 cases are considered true negative. In the 3 other cases, follow-up was not clinically relevant as the patients had the procedure performed on suspicion of relapse of lung cancer, and relapse in mediastinal or neck lymph nodes was found. These cases were considered false negative. 
Fig. 2. Flowchart of patients with a centrally located lung tumor who underwent, in a single session, bronchoscopy, EBUS and EUS-B to obtain a diagnosis of the tumor.

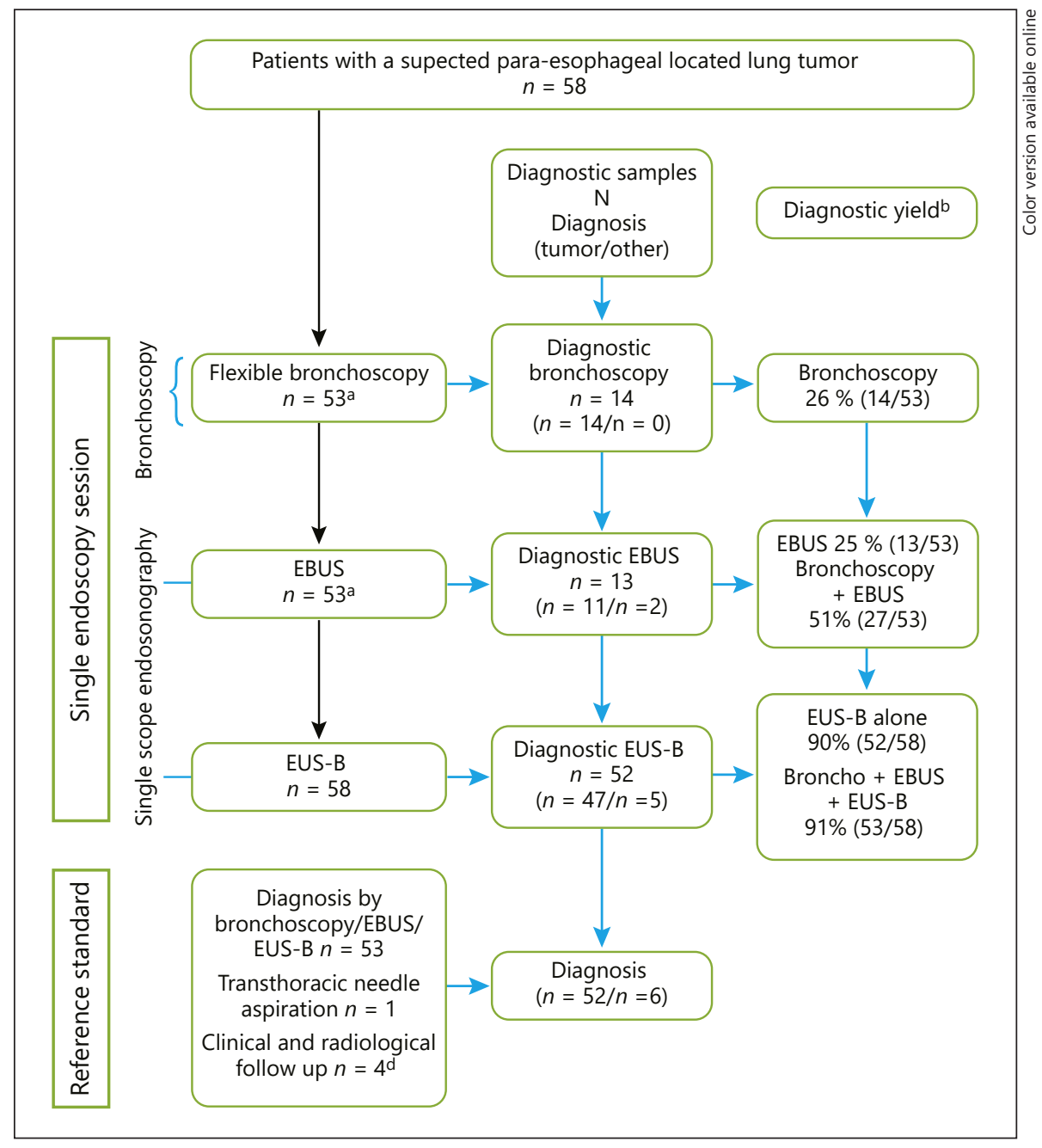

Of the 3 (5\%) inadequate samples, 2 cases had diagnostic adequate samples performed with bronchoscopy or ultrasound-guided transthoracic needle aspiration in the same session as the EUS-B that showed malignancy. One lesion was followed with $\mathrm{CT}$ and showed regression of the lesion in 6 months. All 3 cases were considered false negative. Thus, a total of 52 lesions were considered as malignant.

Transthoracic needle aspiration biopsy was performed and was ultrasound guided in the same session as endoscopic procedures in 4 (7\%) cases to obtain the final diagnosis; in 1 of these, the diagnosis was only established with transthoracic needle aspiration biopsy.

The sensitivity of EUS-B for diagnosing any malignancy was $90 \%$ in this very selected population.
EUS-B-FNA for the Diagnosis of Paraesophageally Located Lung Lesions

\section{Discussion}

In this study, we evaluated the largest series $(n=58)$ of patients who underwent an EUS-B-FNA for diagnosing a centrally located lung tumor adjacent to the esophagus. We found that EUS-B is safe and has a high diagnostic yield and sensitivity (90\%) for diagnosing malignancy. Adding EUS-B to a previous nondiagnostic bronchoscopy and EBUS raised the diagnostic yield from 51 to $91 \%$.

Only one report described EUS-B for diagnosing centrally located lung tumors. Steinfort et al. [17] showed in a small selected cohort study that in 26 out of 27 patients EUS-B was diagnostic. In this study, 10 lesions were inaccessible for bronchoscopic sampling and 9 lesions were inaccessible for EBUS-TBNA. Diagnoses were obtained in predominantly upper lobes and 1 pneumothorax occurred. The current study shows that lung tumors located 
in all different lobes can be visualized and biopsied safely with EUS-B.

In a systematic review and meta-analysis for diagnosing intrapulmonary lung tumors by EUS using the gastrointestinal endoscope, Korevaar et al. [11] showed an average yield of 0.90 and an average sensitivity of 0.92 . The complication rate was low with $2 \%$. Our findings show similar results, indicating that tumor sampling by EUS-B lead to similar results as the conventional EUS scope. The potential advantages of the gastrointestinal EUS scope are the following: the larger overview $\left(120-180^{\circ}\right.$ depended on the scope manufacturer vs. $60^{\circ}$ visualization of EBUS), the needle length (10 vs. $6 \mathrm{~cm}$ ), the slightly superior ultrasound quality (due to the increased amount of ultrasound crystals of the EUS transducer), and the increased stiffness of the scope. However, in clinical practice, all of the abovementioned items were rated not significant and not a limiting factor for the diagnostic yield of the EUS-B approach.

An advantage of using EUS-B instead of convention gastrointestinal EUS scope for diagnosing lung tumors is that the whole diagnostic and staging procedure can be performed in a single endoscopy session performed by one operator. In our study, $91 \%$ of the patients also underwent a conventional bronchoscopy and EBUS in the same session and mediastinal staging was performed in this single session. However, in $45 \%$ of the patients, the lung tumor was only detected and biopsied by EUS-B showing the benefit of this transesophageal approach.

An additional advantage of EUS-(B) is that it can be helpful in assessing mediastinal tumor invasion (T4). We have shown that in patients with paraesophageally located lung tumor the EUS assessment (presents absences of mediastinal tumor invasion) has added important value to the CT scan of the chest [21].

Although CT-guided transthoracic needle aspirations for centrally located parabronchial lesions can be technically undertaken, the significant drawbacks are a high risk of pneumothorax and hemoptysis [16]. In addition, the diagnostic yield is lower than for peripheral lesions [10, $22,23]$.
It should be noted that several limitations apply to this study. First the retrospective character of this study means that there is a large bias of patient selection and the data should be interpreted accordingly. Second, this study analyzed data from 3 centers with expert EUS-B operators. It remains unclear if less experienced endoscopists can achieve similar results. Third visualization of intrapulmonary tumors from the esophagus is only possible if the tumor is located near or adjacent to the esophagus. The maximum distance from the tumor to the esophagus is unknown. In the present study, virtually all tumors were located adjacent to the esophagus. Whether a specific air space between the wall of the esophagus and the lung tumor (as seen on the CT) still allows lung tumor detection by EUS-B needs to be investigated. As the esophagus is located in the left posterior chest, this most often applies to central located leftsided tumors.

Future studies should include larger cohorts in a prospective consecutive design. Our results provide further support that pulmonologists staging lung cancer should be trained in EUS-B-FNA [10].

\section{Conclusion}

EUS-B-FNA is a feasible and safe technique for diagnosing centrally located intrapulmonary tumors that are located near or adjacent to the esophagus. EUS-B should be considered in the same endoscopy session following a nondiagnostic bronchoscopy and EBUS.

\section{Disclosure Statement}

The authors declare that they have no conflicts of interest with respect to the content of this paper. This research did not receive any specific grant from funding agencies in the public, commercial or not-for-profit sectors.

\section{References}

1 Global Burden of Disease Cancer Collaboration: Global, regional, and national cancer incidence, mortality, years of life lost, years lived with disability, and disability-adjusted lifeyears for 32 cancer groups, 1990 to 2015: a systematic analysis for the global burden of disease study. JAMA Oncol 2017;3:524-548.

2 Rivera MP, Mehta AC, Wahidi MM: Establishing the diagnosis of lung cancer: Diagno- sis and management of lung cancer, 3rd ed: American College of Chest Physicians evidence-based clinical practice guidelines. Chest 2013;143(5 Suppl):e142S-e165S.

3 Du Rand IA, Barber PV, Goldring J, Lewis RA, Mandal S, Munavvar M, et al: British Thoracic Society guideline for advanced diagnostic and therapeutic flexible bronchoscopy in adults. Thorax 2011;66:iiil-iii21.
4 Popovich J, Kvale PA, Eichenhorn MS, Radke JR, Ohorodnik JM, Fine G: Diagnostic accuracy of multiple biopsies from flexible fiberoptic bronchoscopy. Am Rev Respir Dis 1982; 125:521-523.

5 Mazzone P, Jain P, Arroliga AC, Matthay RA: Bronchoscopy and needle biopsy techniques for diagnosis and staging of lung cancer. Clin Chest Med 2002;23:137-158, ix. 
6 Cox ID, Bagg LR, Russell NJ, Turner MJ: Relationship of radiologic position to the diagnostic yield of fiberoptic bronchoscopy in bronchial carcinoma. Chest 1984;85:519522.

7 Dhillon SS, Harris K: Bronchoscopy for the diagnosis of peripheral lung lesions. J Thorac Dis 2017;9:S1047-S1058.

8 Yu K-L, Tsai T-H, Ho C-C, Liao W-Y, Lin C-K, Hsu C-L, et al: The value of radial endobronchial ultrasound-guided bronchial brushing in peripheral non-squamous nonsmall cell lung cancer. Sci Rep 2018;8:5837.

9 Annema JT, Veseliç M, Rabe KF: EUS-guided FNA of centrally located lung tumours following a non-diagnostic bronchoscopy. Lung Cancer 2005;48:357-361.

10 Khan SL, Haris M, Diver S, Miller B, Munavvar M: P70 can endobronchial ultrasound (EBUS) guided transbronchial needle aspiration (TBNA) be used as a first line investigation in the diagnosis of central lung parenchymal lesions? Thorax 2012;67:A94.

11 Korevaar DA, Colella S, Spijker R, Bossuyt PM, Konge L, Clementsen PF, et al: Esophageal endosonography for the diagnosis of intrapulmonary tumors: a systematic review and meta-analysis. Respiration 2017;93:126137.

12 Herth FJF, Krasnik M, Kahn N, Eberhardt R, Ernst A: Combined endoscopic-endobronchial ultrasound-guided fine-needle aspiration of mediastinal lymph nodes through a single bronchoscope in 150 patients with suspected lung cancer. Chest 2010;138:790-794.
13 Hwangbo B, Lee HS, Lee GK, Lim KY, Lee SH, Kim HY, et al: Transoesophageal needle aspiration using a convex probe ultrasonic bronchoscope. Respirology 2009;14:843-849.

14 Hwangbo B, Lee GK, Lee HS, Lim KY, Lee SH, Kim HY, et al: Transbronchial and transesophageal fine-needle aspiration using an ultrasound bronchoscope in mediastinal staging of potentially operable lung cancer. Chest 2010;138:795-802.

15 Wimaleswaran H, Farmer MW, Irving LB, Jennings BR, Steinfort DP: Pulmonologistperformed transoesophageal sampling for lung cancer staging using an endobronchial ultrasound video-bronchoscope: an Australian experience. Intern Med J 2017;47:205210.

16 Vilmann P, Frost Clementsen P, Colella S, Siemsen M, De Leyn P, Dumonceau J-M, et al: Combined endobronchial and oesophageal endosonography for the diagnosis and staging of lung cancer. European Society of Gastrointestinal Endoscopy (ESGE) Guideline, in cooperation with the European Respiratory Society (ERS) and the European Society of Thoracic Surgeons (ESTS). Eur Respir J 2015; 46:40-60.

17 Steinfort DP, Farmer MW, Irving LB, Jennings BR: Pulmonologist-performed peresophageal needle aspiration of parenchymal lung lesions using an EBUS bronchoscope. J Bronchology Interv Pulmonol 2017;24:117124.
18 Araya T, Demura Y, Kasahara K, Matsuoka H, Yamamura K, Nishitsuji M, et al: Usefulness of transesophageal bronchoscopic ultrasound-guided fine-needle aspiration in the pathologic and molecular diagnosis of lung cancer lesions adjacent to the esophagus. J Bronchology Interv Pulmonol 2013;20:121126

19 Davoudi M, Colt HG, Osann KE, Lamb CR, Mullon JJ: Endobronchial ultrasound skills and tasks assessment tool: assessing the validity evidence for a test of endobronchial ultrasound-guided transbronchial needle aspiration operator skill. Am J Respir Crit Care Med 2012;186:773-779.

20 Konge L, Vilmann P, Clementsen P, Annema JT, Ringsted C: Reliable and valid assessment of competence in endoscopic ultrasonography and fine-needle aspiration for mediastinal staging of non-small cell lung cancer. Endoscopy 2012;44:928-933.

21 Kuijvenhoven JC, Crombag L, Breen DP, van den Berk I, Versteegh MIM, Braun J, et al: Esophageal ultrasound (EUS) assessment of T4 status in NSCLC patients. Lung Cancer 2017;114:50-55.

22 Heerink WJ, de Bock GH, de Jonge GJ, Groen HJM, Vliegenthart R, Oudkerk M: Complication rates of CT-guided transthoracic lung biopsy: meta-analysis. Eur Radiol 2017;27:138148

23 Boskovic T, Stanic J, Pena-Karan S, Zarogoulidis P, Drevelegas K, Katsikogiannis N, et al: Pneumothorax after transthoracic needle biopsy of lung lesions under CT guidance. J Thorac Dis 2014;6. 